\title{
Quantitative Analysis and Safety Issues of Nanotechnology in Healthcare Research
}

Wellington Pham*

Vanderbilt University School of Medicine, USA

It will be difficult to achieve a smooth translation for nanotechnology if universal quantitative analytical techniques have not been fully implemented in the characterization process.

In the past decade, nanotechnology has made the headlines on numerous occasions. Interestingly, it seems those nano-sized materials have garnered far more attention than their tiny size would seem to justify in healthcare research, including approaches for personalized medicine. At the same time, however, precautious issues have also been raised as well, due to the implication of nanoscale physics on environment, health and safety for producers to end users.

What, then, makes the development of nanotechnology such a controversial issue? No doubt, nanomaterials certainly offer promises. Yet health experts are concerned that this area of research has grown so fast that without appropriate regulation, unintended consequences will outweighs the potential economic and health benefits. Today, with more than 1,000 nanoscale materials available commercially (Nanotechnology and Environmental, Health, and Safety: Issues for Consideration. Congressional Research Service Report 2011), it is vital to protect the public from harm.

Quite obviously, there are lessons to be learned from previous instances of pharmaceutical development fiascos, such as the case of Thalidomide. But while Thalidomide was produced by a single company; today's nanomaterials are being manufactured by a variety of firms and laboratories worldwide. Indeed, a single component could be contributed by any of a number of global raw materials suppliers, thus making identification of the source of a problem and pinpointing the responsible party a much lengthier and difficult process than one might believe.

In healthcare research, nanoscale particles represent a burgeoning area of research. This technique has the potential to offer fundamental changes in diagnostics and novel interventions for disease treatment. Naturally, those nanomaterials are nonspecific for biological study. If specific ligands are incorporated in the nanoparticles, then the result would be a highly specific and targeted probe. In fact, all these advances in nanotechnology are possible only because the inherent multivalency of nanoparticles offers myriad surface modifications for molecular imaging, drug delivery, vaccine delay release applications and more. However, the process of colloidal surface modification and characterization are devious. For instance, we must consider the large body of work from relevant literature which describes that nanomaterials having been mixed with the ligands in the presence of an activating reagent, such as dicyclohexylcarbodiimide or a water soluble version of 1-ethyl-3-(3-dimethylaminopropyl)carbodiimide, and assuming the products are present after dialysis. In reality, the process of chemical coupling under aqueous conditions is insufficient. Intense characterization via analytical chemistry is necessary not only to determine if the desired products have been obtained, but also quantification of the labeled products is crucial and should be used as a parameter to ensure reproducibility and safety.

It is not easy to overcome current practices since quantification of nanomaterials is expensive. After all, not every laboratory is equipped with the sophisticated analytical instruments needed to perform the work. Further, researchers prefer quick turnovers of their work through publications. Still, such considerations cannot be used as excuses.

Characterization of the chemical product is not a matter of if, but rather when. Yet regardless of the answer, one thing is certain: if nanotechnology is being groomed for human application, then appropriate characterization of the chemistry at the beginning of the conceptual processes may prove beneficial overtime, particularly with regard to GMP and IND processes. After all, in the face of a decade spent validating a spectrum of cell and animal models, the failure of the materials to meet FDA requirements because of lack of quantitative analysis would be intolerable.

Several scientific panels have already begun deliberating the safety issues of nanotechnology in healthcare. Among the important topics of discussion is the need to safeguard of human health via strenuous implementation of analytical techniques to characterize nanoproducts. The lack of such an approach would hinder the translation of this promising science from bench to bedside. With that in mind, each of us has a major role to play shares the responsibility to emphasize this important issue at all levels. Remaining passive or even silent about this challenge would only restrict the contributions nanotechnology could make in biomedical research. Clearly, the need for quantitative analysis as a forerunner to the impeding translation process cannot be marginalized.

*Corresponding author: Wellington Pham, $\mathrm{PhD}$, Biomedical Engineering, Vanderbilt University, USA, Tel: +615-936-7621; E-mail: wellington.pham@Vanderbilt.Edu

Received September 21, 2012; Accepted September 21, 2012; Published September 24, 2012

Citation: Pham W (2012) Quantitative Analysis and Safety Issues of Nanotechnology in Healthcare Research. J Mol Biomark Diagn 3:e111. doi:10.4172/2155-9929.1000e111

Copyright: (C) 2012 Pham W. This is an open-access article distributed under the terms of the Creative Commons Attribution License, which permits unrestricted use, distribution, and reproduction in any medium, provided the original author and source are credited 